EPJ Web of Conferences 35, 02002 (2012)

DOI: 10.1051/epjconf/20123502002

(C) Owned by the authors, published by EDP Sciences, 2012

\title{
Application of Nuclear Microprobes towards Understanding Complex Ore Geo-electrochemistry
}

\author{
J.S.Laird ${ }^{1,2,3}$, R.Szymanski ${ }^{3}$, R.Large ${ }^{2}$ and C.G.Ryan ${ }^{1,2,3}$ \\ ${ }^{1}$ CSIRO, Earth Science and Resource Engineering, Clayton, Victoria, Australia \\ ${ }^{2}$ Centre of Excellence in Ore Deposits (CODES), University of Tasmania, Hobart, Tasmania, Australia \\ ${ }^{3}$ School of Physics, University of Melbourne, Parkville 3010, Victoria, Australia
}

\begin{abstract}
We report on recent development on the CSIRO Nuclear Microprobe (NMP) towards catering for long exposure mapping required for large area scanning. A new data collection system based on Labview FPGA highly co-ordinated with beam transport sits at the heart of the upgrade. These upgrades are discussed and an example of the systems use for $\mu$-Particle Induced X-ray Emission (PIXE) analysis in the area of complex ore geo-electrochemistry is briefly described.
\end{abstract}

\section{Introduction}

A Nuclear Microprobe (NMP) attached to the University of Melbourne $5 \mathrm{MeV}$ Pelletron accelerator allows finely focused $\mu$-Particle Induced X-ray Emission (PIXE) imaging of trace, minor and major elements across most of the periodic table [1]. The unique focusing system of the CSIRO beamline routinely provides $1-2 \mu \mathrm{m}$ spatial resolution with average ppm level sensitivities for elements of interest to trace geochemistry. Numerous other methods also available on the CSIRO NMP including Ionoluminescence (IL) microscopy [2], Ion Beam Induced Current [3], Rutherford Backscattering and Particle Induced Gamma-ray Emission (PIGE). These enhance elemental mapping by providing coincident chemical information, structural morphology and light element concentrations, respectively.

In recent times however there has been an increased impetus on increasing scan size whilst maintaining the maximum spatial resolution. This requires optimisation of the data collection system and importantly, the ability to collect over extended periods of time i.e reliable $24 \mathrm{hr}$ operation. In this paper we report on the development of a new data collection platform stable over many days and able to provide real-time feedback for automating beam transport along the microbeam line.

\section{Micro-PIXE on the CSIRO NMP}

\subsection{Labview FPGA Data Collection}

A Field Programmable Gate Array (FPGA) based data collection system called Microdaq has been developed for the CSIRO Nuclear Microprobe (NMP) and interlinked with new XYZ stage, object and divergence slit control systems as illustrated in Fig. 1 below.

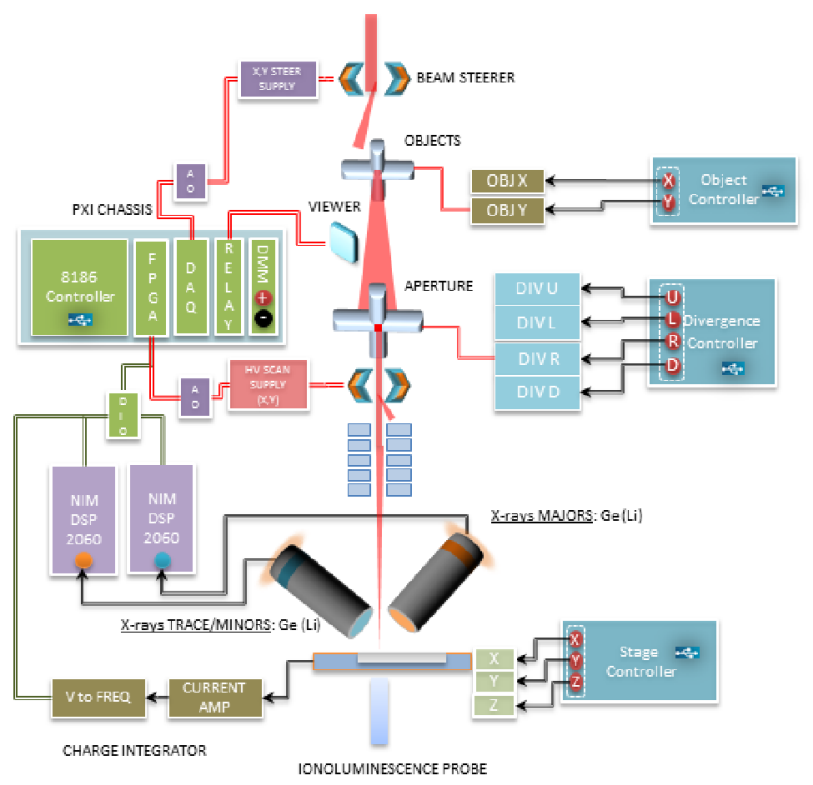

Fig. 1. Overall schematic of the new beam transport and data collection system for the CSIRO NMP. 
At the core of the system is a National Instruments PXI chassis with a real-time controller as well as numerous modules including a RIO FPGA, a DAQ card for general non-deterministic acquisitions including beam steering, and a multiplexer/relay module for controlling assorted devices such as pneumatics associated with the object and divergence monitors etc.

Arcus technology stepper motors and drivers are used for controlling both object slits, four divergence slits as well as the $\mathrm{XYZ}$ target stage. At present a camera positioned at the beam viewer position (prior to the divergence limiting slits) records the beam position and allows automatic steering to optimise beam current in the target chamber. This requires a beam stop. In future, a Femto current amplifier multiplexed to each divergence slit will in-situ monitor beam centering on the ion optical axis without interfering with data collection.

The Labview based system caters for numerous detector signals including hyperspectral IL data ionoluminescence data as well as NIM ADC data with full dead-time correction and pixel charge normalisation. Typically dual PIXE channels collect both major and minor/trace element maps as shown in the figure. The system was designed to accommodate rapid prototyping of new experimental configurations. For example, shape scanning for Proton Beam Writing (PBW) has also been catered for. The entire system is tightly interlinked to allow long term PIXE experiments some of which have lasted up to a continuous 72 hours. A capture of the front panel is shown in Fig. 2.

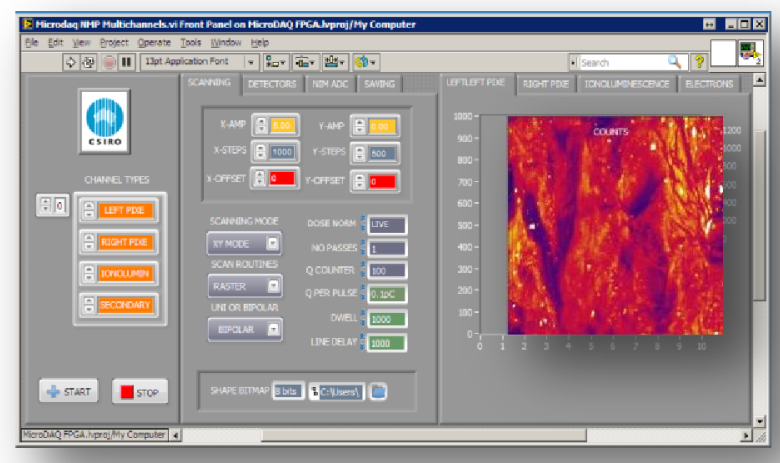

Fig. 2. Front panel capture of the Labview FPGA data collection system for the CSIRO NMP.

\section{Example Data}

An example of a recent data-set centred around the correlation of elemental heterogeneity with the electrical properties of a prite mineral from Otago, New Zealand is now described. Detailed analysis of the data is to be made elsewhere [4]. Shown in Fig. 3 is the total PIXE spectrum for a $4 \mathrm{~mm} \times 1.5 \mathrm{~mm}$ scan of $3 \mathrm{MeV}$ protons with an accumulated charge of around $\sim 200 \mu \mathrm{C}(50 \mathrm{pC}$ per pixel). Fitting to the spectrum using the GeoPIXE software suite
[5] including background subtraction due to pile-up and escape peaks is also shown. Using the dynamic analysis matrix determined from this fit, images of elemental concentration were then generated as shown in Fig. 4. The entire region subjected to Laser Beam Induced Current analysis (LBIC) [4]. All of the elements shown had large concentrations compared to the Minimum Detection Limit (MDL) expected for this accumulated charge ( $\mathrm{ppm}$ to sub-ppm range for most elements beyond $\mathrm{Fe})$.

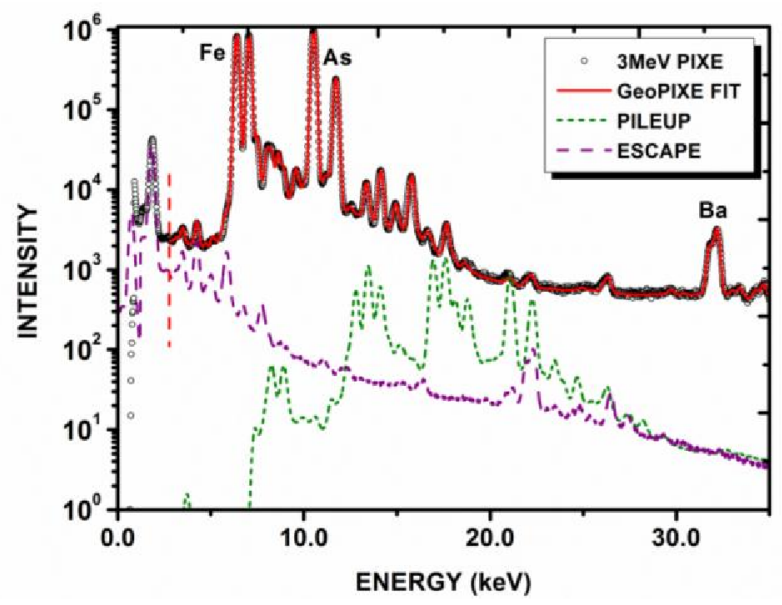

Fig. 3. Total PIXE spectrum and GeoPIXE fit including background contributions from pile-up and Ge escape peaks.

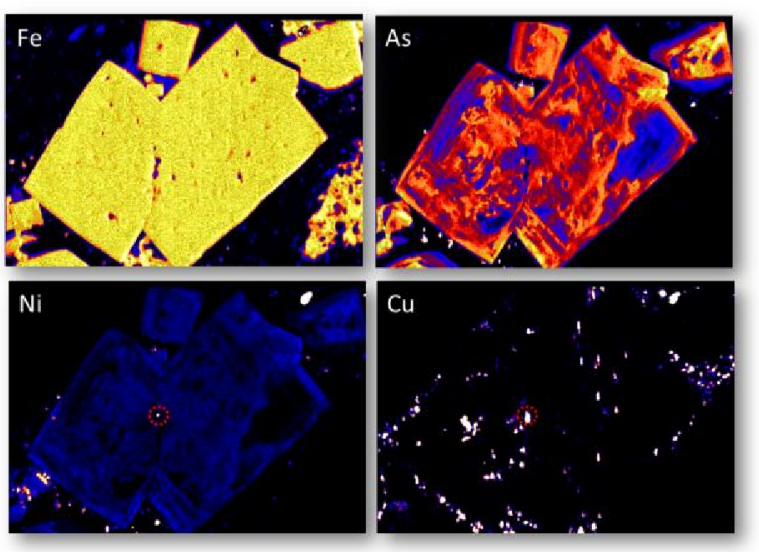

Fig. 4. A collection of PIXE images of the main and minor elements in one Otago pyrite grain.

\section{Conclusion}

The new Microdaq data collection system has been briefly described and illustrated on a pyrite mineral from Otago, N.Z. The system is still undergoing gradual improvements including the addition of extra channels as deemed necessary. The microbeam facility is now running 24 hrs a day and producing high quality PIXE data for the geological and material sciences. Also planned for future operations is a PXI controlled system for real-time control of the accelerator and ancillary systems. 


\section{Acknowledgements}

The authors would like to gratefully acknowledge a large Australian Education Infrastructure Fund (EIF) grant for some of this work.

\section{References}

1. Ryan, C.G., et al., The new CSIRO-GEMOC nuclear microprobe: First results, performance and recent applications. Nuclear Instruments \& Methods in Physics Research Section B-Beam Interactions with Materials and Atoms, 2001. 181: p. 12-19.

2. Laird, J.S., et al., Hyper-spectral ionoluminescence system for minerals and fluid inclusions. Nuclear Instruments and Methods in Physics Research Section B: Beam Interactions with Materials and Atoms, 2011. 269(20): p. 2244-2250.

3. Laird, J.S., et al., Impurity mapping in sulphide minerals using Time-resolved Ion Beam Induced Current imaging. Nuclear Instruments and Methods in Physics Research Section B: Beam Interactions with Materials and Atoms, 2010. 268(11-12): p. 1903-1910.

4. Laird, J.S., et al., Internal Electric Fields in Arsenian Pyrite associated with Impurity and Mixed Sulfide Heterogeneity. American Mineralogist, 2012. To Be Submitted.

5. Ryan, C.G., et al., QUANTITATIVE PIXE MICROANALYSIS OF GEOLOGICAL MATERIAL USING THE CSIRO PROTON MICROPROBE. Nuclear Instruments \& Methods in Physics Research Section B-Beam Interactions with Materials and Atoms, 1990. 47(1): p. 55-71. 